\title{
Fate of Myelin Lipids during Degeneration and Regeneration of Peripheral Nerve: An Autoradiographic Study
}

\author{
Jeffry F. Goodrum, ${ }^{1,2}$ Todd Earnhardt, ${ }^{1}$ Nelson Goines, ${ }^{1}$ and Thomas W. Bouldin ${ }^{2}$ \\ 'Brain and Development Research Center and 'Department of Pathology, University of North Carolina at Chapel Hill, \\ Chapel Hill, North Carolina 27599
}

Four weeks after labeling myelin lipids with an intraneural injection of ${ }^{3} \mathrm{H}$-acetate, sciatic nerves were crushed, and the distribution of radiolabeled myelin lipids was followed by autoradiography from $1 \mathrm{~d}$ to 10 weeks later. Just prior to crush, silver grains were localized to the myelin sheath. Three days after crush, axons were degenerating and myelin sheaths were breaking down; silver grains appeared over lipid droplets within Schwann cells, fibroblasts, and macrophages. One week after crush the basal-lamina-delimited Schwann-cell tubes (Büngner bands) contained myelin debris, and some tubes already contained regenerating axons. Schwann cells were often displaced to the periphery of the tubes by phagocytes containing heavily labeled myelin debris; extratubal macrophages within the endoneurium contained labeled lipid droplets but no myelin debris. Two weeks after nerve crush silver grains were associated with newly formed myelin around regenerating axons. Many extratubal endoneurial macrophages now contained labeled myelin debris and lipid droplets. By 3 weeks myelination of regenerating axons was advanced, and the myelin sheaths were well labeled. Extratubal macrophages had become the major labeled structure within the nerve because they contained large amounts of labeled myelin debris and lipid droplets. From 4 to 10 weeks after nerve crush the new myelin sheaths continued to thicken and to be well labeled. Debris-laden extratubal macrophages remained the major site of labeled material within the endoneurium. Our results confirm that there is reutilization of myelin cholesterol by Schwann cells to form new myelin, and indicate that some lipid catabolism takes place in Schwann cells and endoneurial fibroblasts prior to infiltration of the nerve by macrophages. However, most of the myelin debris is phagocytized by macrophages within 1-2 weeks following nerve injury. These debris-laden macrophages persist within the nerve for many weeks, indicating that much of the salvaged cholesterol is not reutilized for myelin regeneration.

[Key words: autoradiography, cholesterol, lipoproteins, macrophages, myelin, peripheral nerve, Wallerian degeneration]

\footnotetext{
Received Apr. 30, 1993; revised July 8, 1993; accepted July 15, 1993.

This study was supported by U.S. Public Health Service Grant ES-01104 in a center receiving core support from HD-03110.

Correspondence should be addressed to Dr. Jeffry F. Goodrum, Department of Pathology, CB\# 7525, 409 Brinkhous-Bullitt Building, University of North Carolina at Chapel Hill, Chapel Hill, NC 27599.

Copyright (c) 1994 Society for Neuroscience $0270-6474 / 94 / 140357-11 \$ 05.00 / 0$
}

The relative roles of the Schwann cell and the macrophage in the early and late catabolism of degenerating myelin in the PNS are still controversial. Studies of nerve segments degenerating in situations that exclude macrophages suggest that the macrophage has an essential and exclusive role in myelin degradation (Beuche and Friede, 1984; Crang and Blakemore, 1986). Contrariwise, Boyles et al. (1989) concluded from a morphologic study of the cell types containing myelin debris and lipid droplets following nerve crush that the Schwann cell is solely responsible for the absorption and degradation of degenerating myelin. Staking out the middle ground, Stoll et al. (1989) concluded from a morphologic study of the immunochemically defined cell types containing myelin debris and lipid droplets that the Schwann cell participates in the early degradation of myelin, but that the macrophage is responsible for most of the myelin degradation in peripheral ncrve. The rolc of the cndoneurial fibroblast has received less attention, but it too has been implicated in the degradation of myelin (e.g., Shubert and Friede, 1981).

Recent biochemical studies of myelin degeneration have focused on the fate of myelin lipids. Breakdown of myelin phospholipids and formation of cholesteryl esters are very early events following nerve crush, and still occur when invasion of the nerve by macrophages is prevented (White et al., 1989). The fatty acids released during the breakdown of phospholipids are postulated to be reutilized locally in the synthesis of glycerolipids (White et al., 1989). At least a portion of the myelin cholesterol in peripheral nerve is also retained within the nerve following a nerve crush, and is subsequently reutilized for the synthesis of new myelin during regeneration of the nerve (Rawlins et al., 1970, 1972). A putative mechanism for this process of cholestcrol reutilization was provided by the discovery of apolipoprotein $\mathrm{E}$ (apoE) synthesis and secretion in injured nerve (Ignatius et al., 1986; Snipes et al., 1986). It has been hypothesized that endoneurial apoE, functioning in a manner analogous to its role in systemic cholesterol transport, salvages degenerating myelin cholesterol in the form of lipoprotein particles, and then resupplies the cholesterol to myelinating Schwann cells via the low-density lipoprotein receptor. Hematogenously derived macrophages, attracted to the nerve by the degenerating nerve fibers, are the source of the endoneurial apoE, and are postulated to be a storage depot for the salvaged cholesterol (see Mahley, 1988, for review). There is now considerable evidence supporting this hypothesis (Boyles et al., 1989, 1990; Goodrum, 1990, 1991; Gelman et al., 1991), although we have recently shown that apoE is not necessary for fiber regeneration or the local reutilization of cholesterol (Popko et al., 1993). 
Figure 1. EM autoradiograph of an uncrushed sciatic nerve 4 weeks after an intraneural injection of ${ }^{3} \mathrm{H}$-acetate. Myelin sheaths are well labeled. Unstained section; $6800 \times$.

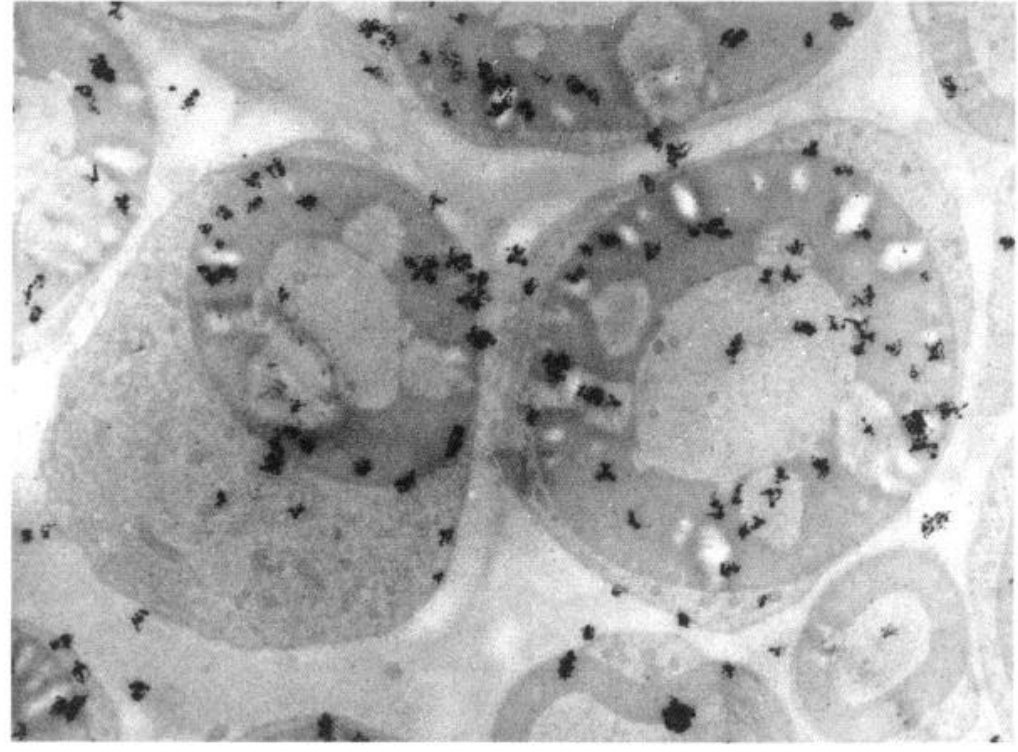

We have followed by electron microscopic (EM) autoradiography the distribution of prelabeled myelin lipids (predominantly cholesterol) from $1 \mathrm{~d}$ to 10 weeks following a nerve crush and the distribution of newly synthesized lipids at $2 \mathrm{~d}$ after nerve crush to test whether these proposed sequences of myelin metabolism during Wallerian degeneration are valid. Our results confirm that there is reutilization of myelin cholesterol by Schwann cells to form new myelin, and indicate that some lipid catabolism takes place in Schwann cells and endoneurial fibroblasts prior to infiltration of the nerve by macrophages. However, most of the myelin debris is phagocytized by macrophages within 1-2 weeks following nerve injury. These debris-laden macrophages persist within the nerve for many weeks, indicating that much of the salvaged cholesterol is not reutilized for myelin regeneration. The implications of these results for the lipoprotein-mediated salvage and reutilization of myelin cholesterol are discussed.

This work was presented in preliminary form at the 69th Annual Meeting of the American Association of Neuropathologists (Goodrum et al., 1993).

\section{Materials and Methods}

\section{Materials}

${ }^{3} \mathrm{H}$-acetate $(500 \mathrm{Ci} / \mathrm{mmol})$ was obtained from ICN Radiochemicals (Irvine, CA). $2-{ }^{3} \mathrm{H}$-glycerol $(15 \mathrm{Ci} / \mathrm{mmol})$ was obtained from ARC (St. Louis, MO). Digitonin and buffer reagents were obtained from Sigma Chemical Co. (St. Louis, MO). Kodak NTB-2 emulsion was from Eastman Kodak (Rochester, NY). Ilford L.4 Nuclear Research emulsion was from Polysciences Inc. (Warrington, PA).

\section{Animal model}

Male Long-Evans rats were bred in our own animal colony from stock originally obtained from the Charles River Breeding Laboratories (Wilmington, MA). The rats were weaned at $20 \mathrm{~d}$ of age, given food and water ad libitum, and kept in solid-bottom plastic cages in a room with constant ambient temperature $\left(21^{\circ} \mathrm{C}\right)$ and cyclic fluorescent light $(12 \mathrm{hr}$ light: $12 \mathrm{hr}$ dark). All animal use procedures were in strict accordance with the NIH Guide for the Care of Laboratory Animals and were approved by the local Animal Use Committee.

Intraneural injection. At $20 \mathrm{~d}$ of age, when the rate of myelination in sciatic nerve is high, newly synthesized nerve lipids were labeled in vivo via intraneural injection of ${ }^{3} \mathrm{H}$-acetate as previously described (Goodrum et al., 1990). Rats $(n=27)$ were anesthetized with an intramuscular injection of ketamine $(87 \mathrm{mg} / \mathrm{kg})$ and xylazine $(13 \mathrm{mg} / \mathrm{kg})$, and the left sciatic nerve was exposed. A hand-drawn glass micropipette (with a tip diameter of $25-100 \mu \mathrm{m}$ ) held in a micromanipulator was inserted 3-5 $\mathrm{mm}$ into the nerve just distal to the sciatic notch. One microliter of sterile saline solution containing $100 \mu \mathrm{Ci}$ of ${ }^{3} \mathrm{H}$-acetate was injected using gentle back-pressure from an air-filled syringe.

Nerve crush. Four weeks after the intraneural injection of acetate most nonmyelin lipids are no longer labeled (Goodrum et al., 1990). At this time, the 48-d-old rats were anesthetized with pentobarbital and the left sciatic nerve exposed in the upper thigh region. The nerve was crushed with jeweler's forceps for $30 \mathrm{sec}$ just distal to the sciatic notch and proximal to the intraneural injection site. From $1 \mathrm{~d}$ to 10 weeks after the nerve crush, rats ( $n=3$ for each time point) were anesthetized and perfusion fixed. Following fixation, segments from the injected sciatic nerves were taken in the region of the injection site and processed for autoradiography as described below.

In vitro incubations. Left sciatic nerves were crushed in a group of 48-d-old rats without prior intraneural injection, as described above. Two days after nerve crush the rats were anesthetized and a $10 \mathrm{~mm}$ segment of the sciatic nerve distal to the crush site was removed and desheathed. Half the segment was incubated with $200 \mu \mathrm{Ci}$ of ${ }^{3} \mathrm{H}$-acetate, and the other half incubated with $200 \mu \mathrm{Ci}$ of $2{ }^{3} \mathrm{H}$-glycerol for $1 \mathrm{hr}$ to label newly synthesized lipids, as previously described (Goodrum et al., 1990). Incorporation of label from acetate represents bulk de novo synthesis of lipids, while incorporation of label from glycerol represents both de novo synthesis and a recycling of fatty acyl moieties from the degradation of glycerolipids. After extensive washing to remove unincorporated label, the nerve segments were immersion fixed and processed for autoradiography as described below.

\section{Morphological methods}

Tissue preparation. Rats were anesthetized as described above and perfused transcardially with $200 \mathrm{ml}$ of $4 \%$ glutaraldehyde in a $0.05 \mathrm{M}$ phosphate buffer ( $\mathrm{pH} 7.4$ ) over a $10-15 \mathrm{~min}$ interval. A $5 \mathrm{~mm}$ segment of the injected left sciatic nerve was excised from the region of the injection and immersion fixed for $24 \mathrm{hr}$ in $4 \%$ glutaraldehyde containing $0.2 \%$ digitonin (to prevent the extraction of cholesterol in subsequent steps) (Napolitano and Scallen, 1969; Scallen and Dietert, 1969). Nerve segments were then postfixed in Dalton's chrome osmium for $1 \mathrm{hr}$, dehydrated rapidly in a series of ice-cold graded acetone solutions to maximize retention of lipids (Gould et al., 1987), and embedded in Spurr's resin. Liquid scintillation spectroscopy of acetone solutions revealed that less than $10 \%$ of the incorporated label was extracted by these procedures.

Autoradiographic methods. Our methods for light microscopic (LM) autoradiography were as previously reported (Goodrum et al., 1990). Briefly, $1-\mu \mathrm{m}$-thick longitudinal and cross sections of plastic-embedded sciatic nerve were placed on clean glass slides and coated with a 1:1 

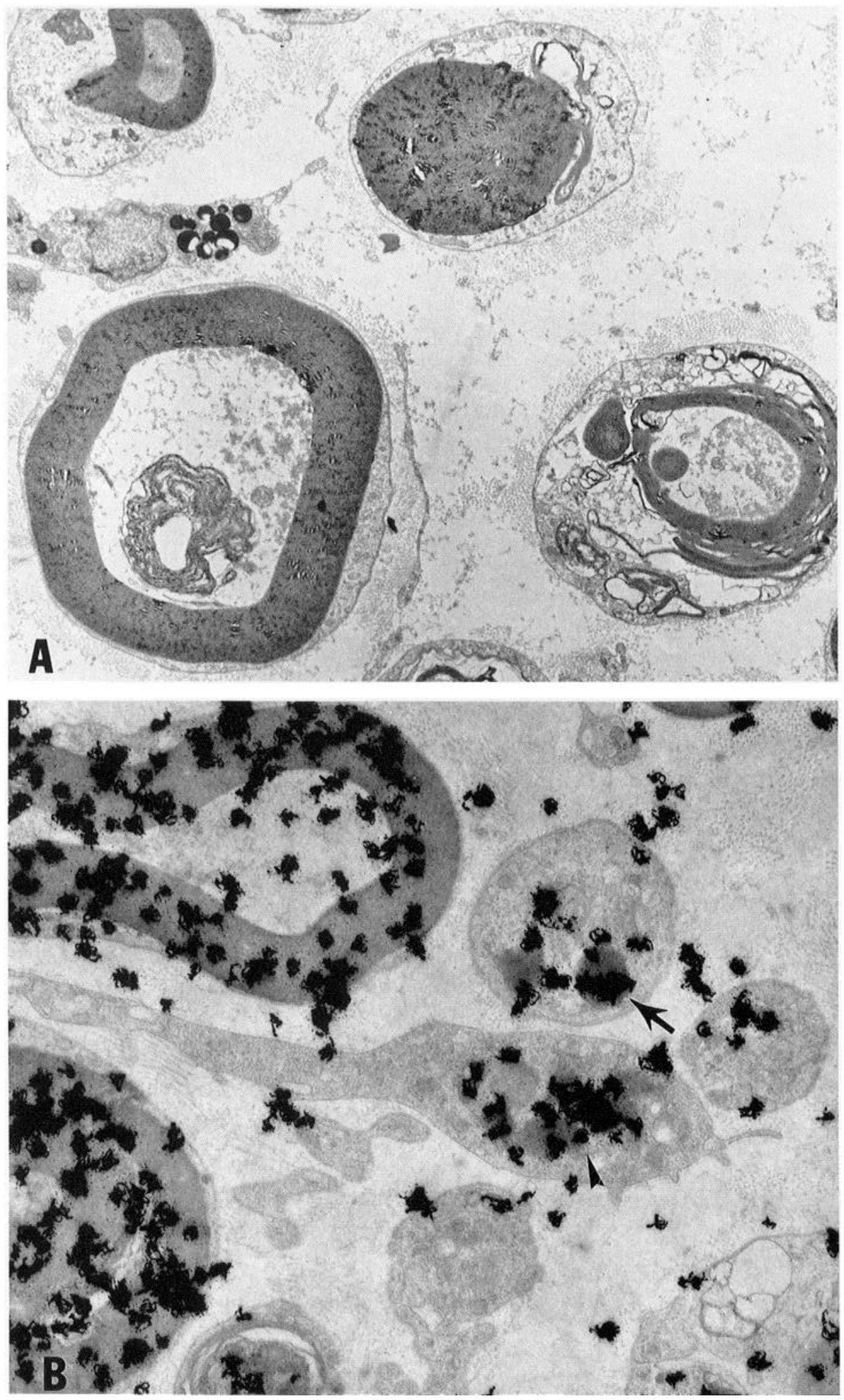

Figure 2. Electron micrograph $(A)$ and EM autoradiograph $(B)$ of sciatic nerve $3 \mathrm{~d}$ after nerve crush. $A$, Axons show granular degeneration. Myelin sheaths are in various stages of degeneration. Stained section; $5900 \times$. B, Degenerating myelin sheaths are well labeled. Lipid droplets within Schwann cells (arrow) and fibroblasts (arrowhead) are also labeled. Unstained section; $8900 \times$. aqueous dilution of Kodak NTB-2 emulsion and then exposed at $4^{\circ} \mathrm{C}$ for $1-10$ weeks. Sections were developed at $20^{\circ} \mathrm{C}$ in Microdol-X developer and then stained with paraphenylenediamine. Twelve sections from each nerve were examined.

EM autoradiography methods were adapted from Salpeter (1981) and were performed as previously described (Goodrum et al., 1990). Ultrathin sections of gold interference color were collected from a water bath onto collodion-coated slides. After drying overnight, all sections were carbon coated. Sections were subsequently hand dipped for $5 \mathrm{sec}$ in a $45^{\circ} \mathrm{C}$ solution $(1 \mathrm{gm} / 5 \mathrm{ml})$ of Ilford L. 4 Nuclear Research emulsion, dried for $2 \mathrm{hr}$, and then stored in light-tight boxes at $4^{\circ} \mathrm{C}$. The sections were developed (1-2 weeks later for the in vitro labeled tissues and 618 weeks later for the in vivo labeled tissues) with D-19 for $2 \mathrm{~min}$ at $20^{\circ} \mathrm{C}$, washed in deionized water $15 \mathrm{sec}$, dipped in $1 \%$ acetic acid stop bath for $15 \mathrm{sec}$, washed $15 \mathrm{sec}$, fixed for $2 \mathrm{~min}$ in fixative, and given two final washes of $30 \mathrm{sec}$ each. After stripping the collodion film and 

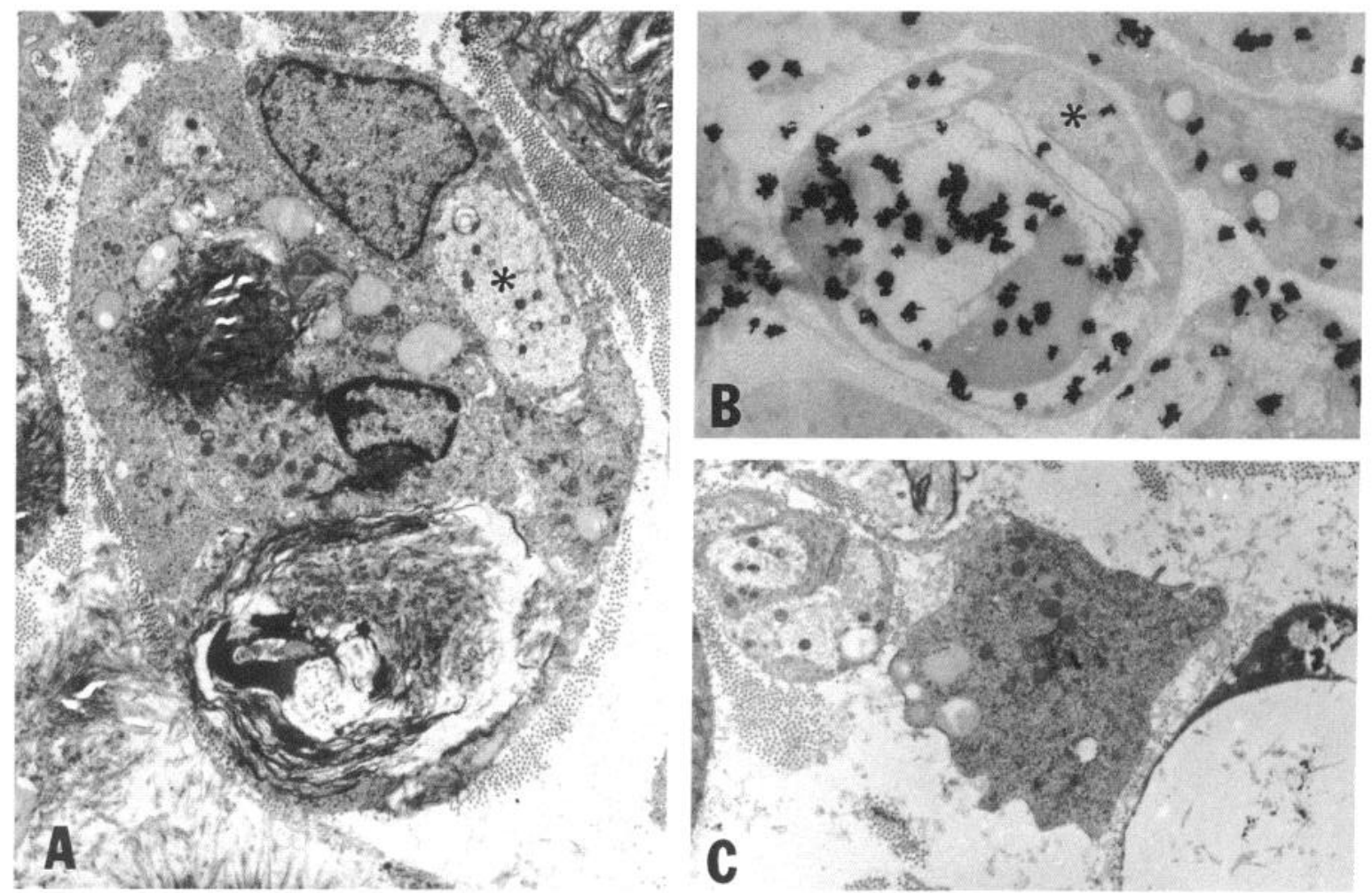

Figure 3. Electron micrographs and EM autoradiograph of sciatic nerve 1 week after nerve crush. $A$, Schwann cell within Schwann-cell tube contains regenerating axon (asterisk). Contiguous, intratubal phagocyte contains myelin debris and lipid droplets. Stained section; $6300 \times$. $B$, EM autoradiograph showing intratubal phagocyte with heavily labeled myelin debris. The phagocyte is partially surrounded by a Schwann cell containing a regenerating axon (asterisk). Unstained section; $6100 \times . C$, Extratubal macrophage contains lipid droplets, but no myelin debris, one week after nerve crush. Stained section; $6500 \times$.

sections off the slides in a deionized water bath, the sections were taken out of the water bath on 200 mesh copper grids, dried, and examined in a Zeiss 10 electron microscope. At least two sections from each nerve were examined. The resolution (HD) of our EM autoradiographic preparations, as defined by Salpeter et al. (1978), was $1500 \AA$.

For conventional electron microscopy, ultrathin sections were stained with uranyl acetate and lead citrate prior to viewing in the electron microscope.

\section{Results}

In vivo labeling

Four weeks following the intraneural injection of ${ }^{3} \mathrm{H}$-acetate and just prior to nerve crush, almost all of the incorporated label in nerve was found in the myelin sheath (Fig. 1). We have previously shown that at this time $60 \%$ of the radiolabel is in cholesterol and $30 \%$ in phospholipids, and that over a 12 week course of nerve degeneration and regeneration, more than $80 \%$ of the total label comes to represent cholesterol as other lipids within the nerve are degraded (Goodrum, 1991).

One day after nerve crush EM revealed early signs of degeneration in some axons, but myelin sheaths appeared normal. Lipid droplets were rarely found in Schwann cells and fibroblasts. LM and EM autoradiography revealed a distribution of grains identical to that found in uncrushed labeled nerves (not shown).

Three days after nerve crush all fibers had granular degeneration of their axons. Some degenerating myelin sheaths still retained their periodicity, but others were breaking up into myelin ovoids (Fig. 2A). Much of the label remained over the myelin, but there was now an increase in the labeling of Schwann- cell cytoplasm, predominantly over lipid droplets (Fig. 2B). Fibroblasts also contained labeled lipid droplets at this time point (Fig. $2 B$ ). The few resident, extratubal endoneurial macrophages present also contained labeled lipid droplets (not shown).

One week after nerve crush, the degenerating fibers consisted of basal-lamina-delimited Schwann-cell tubes (Büngner bands) containing intracellular myelin ovoids and other myelin debris (Fig. $3 A$ ). Some Schwann-cell tubes already contained regenerating axons. Many of the intratubal Schwann cells were displaced to the periphery and partially surrounded phagocytes filled with labeled myelin debris (Fig. $3 A, B$ ). Occasional extratubal macrophages were present at 1 week and contained lipid droplets, but little myelin debris (Fig. $3 C$ ).

Two weeks after nerve crush, regenerated axons surrounded by thin myelin sheaths were abundant. Silver grains were already associated with this newly forming myelin (Fig. 4A). Myelinating regenerating axons and phagocytes containing labeled myelin debris could be found within the same Schwann-cell tube (Fig. $4 B$ ). Extratubal macrophages were now numerous and contained labeled myelin debris as well as labeled lipid droplets (Fig. 4A). Schwann cells and fibroblasts continued to contain labeled lipid droplets (not shown).

Three weeks after nerve crush, myelination of regenerating axons was advanced, and there was no longer any labeled myelin debris within the Schwann-cell tubes. From this time forward to 10 weeks, the distribution of label within the nerve changed little. The myelin sheaths continued to thicken and were well labeled (Fig. 5A,B). Extratubal macrophages contained much myelin debris and lipid droplets and were the major labeled 

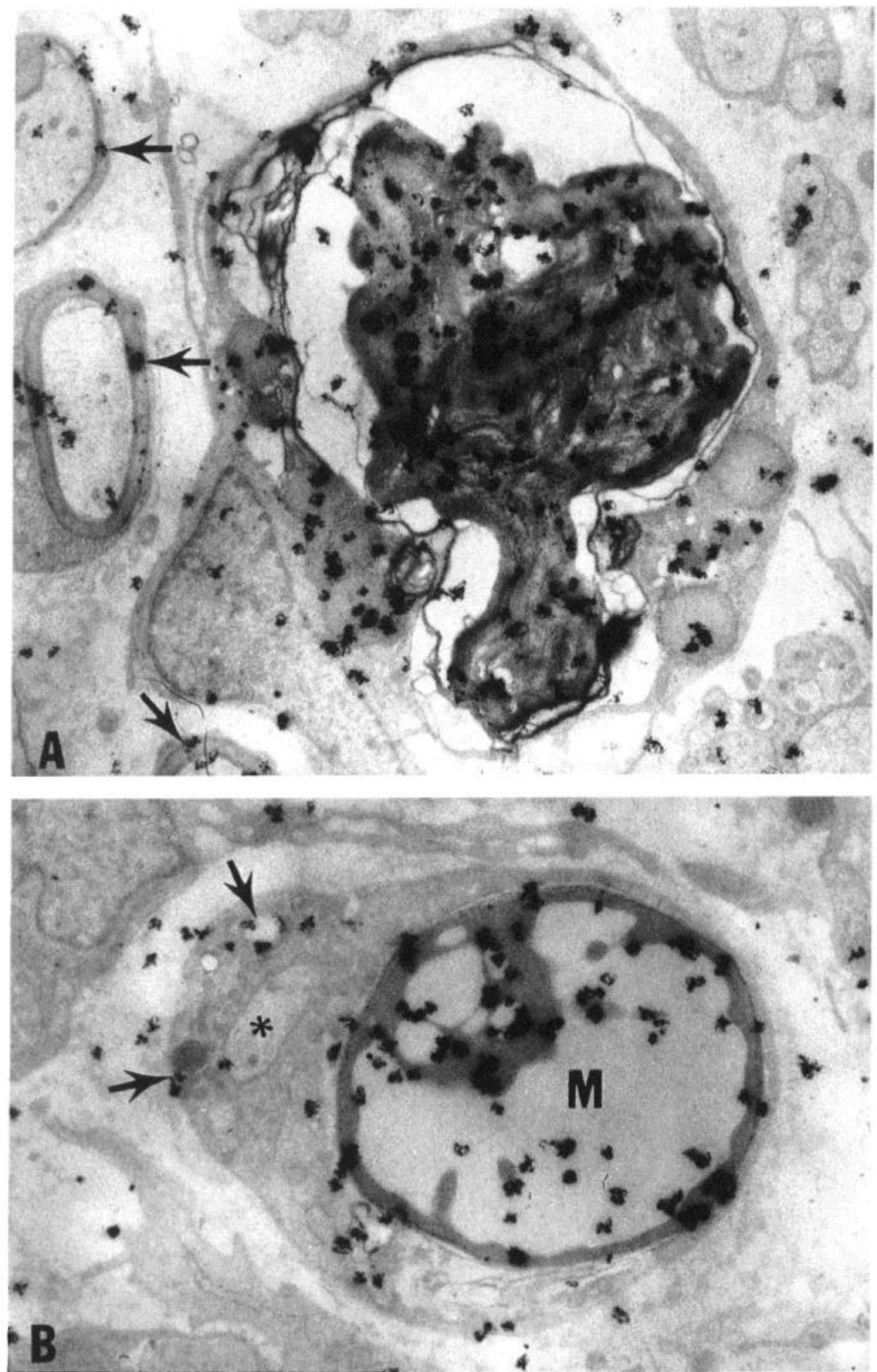

Figure 4. EM autoradiographs of sciatic nerve 2 weeks after nerve crush. $A$, The new myelin covering the regenerating axons is labeled (arrows). An extratubal macrophage in the center of the field is filled with labeled myelin debris and lipid droplets. Unstained; 7500x. $B$, Phagocyte containing labeled myelin debris $(M)$ and a Schwann cell containing a myelinating axon (asterisk) and labeled lipid droplets (arrows) are within the same Schwann-cell tube. Unstained section; $8100 \times$. structure within the nerve (Fig. $5 C$ ). These debris-laden macrophages were within the endoneurium and did not accumulate in the perineurium or epineurium. Lipid droplets in Schwann cells and fibroblasts became progressively less numerous.

Extracellular labeled myelin debris within the endoneurium was noted at four weeks after nerve crush and became more prominent by 10 weeks (Fig. $5 B$ ). It has been suggested that this extracellular debris may represent the detritus of degenerated macrophages (Boyles et al., 1989).

\section{In vitro labeling}

When newly synthesized lipids were labeled by incubating nerve segments with ${ }^{3} \mathrm{H}$-acetate $2 \mathrm{~d}$ after nerve crush, the cytoplasm of Schwann cells and fibroblasts was heavily labeled, including the lipid droplets that were present (Fig. 6A,B). In addition, degenerating myelin became labeled (Fig. $6 A, B$ ). When ${ }^{3} \mathrm{H}$-glycerol was used as the precursor, there was a preferential labeling of the lipid droplets within fibroblasts and Schwann cells; degenerating myelin was also lightly labeled (Fig. $7 A, B$ ).

\section{Discussion}

Myelin catabolism prior to macrophage infiltration

The early appearance of lipid droplets in Schwann cells during Wallerian degeneration has traditionally been interpreted as indicative of a catabolic event in which the Schwann cell is involved in the initial degradation of its own myelin (e.g., Boyles 


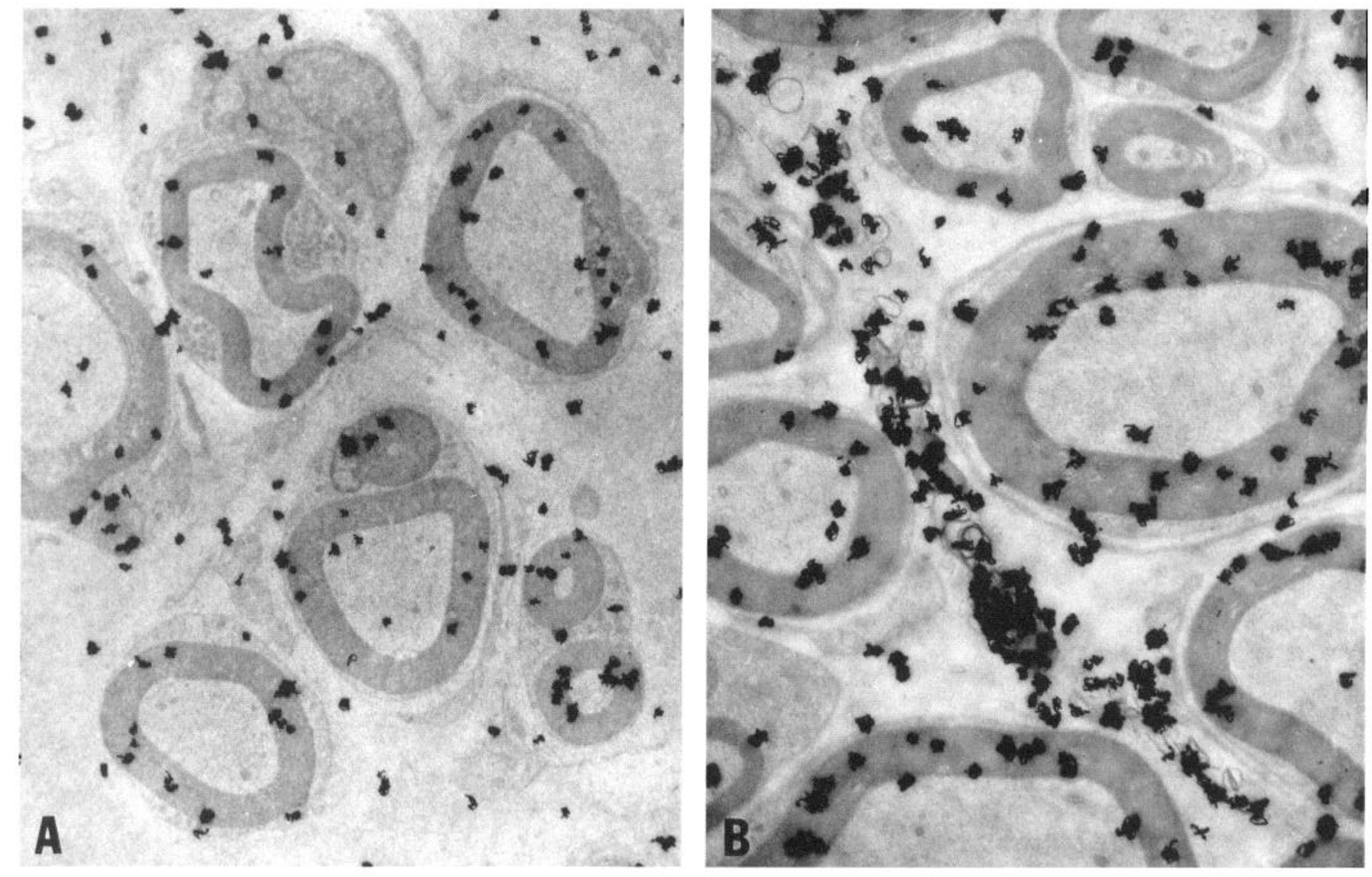

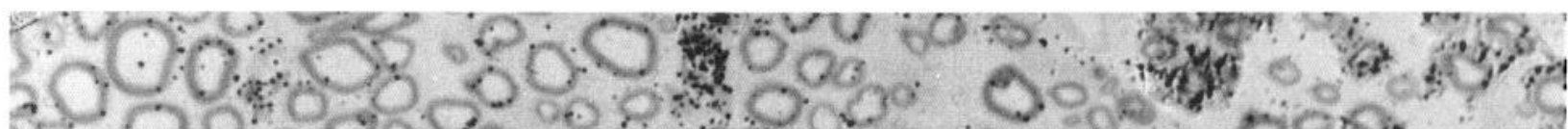

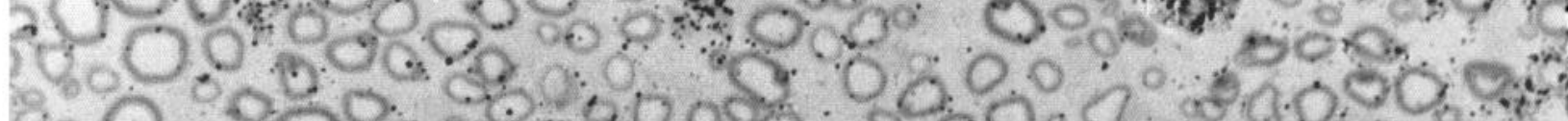

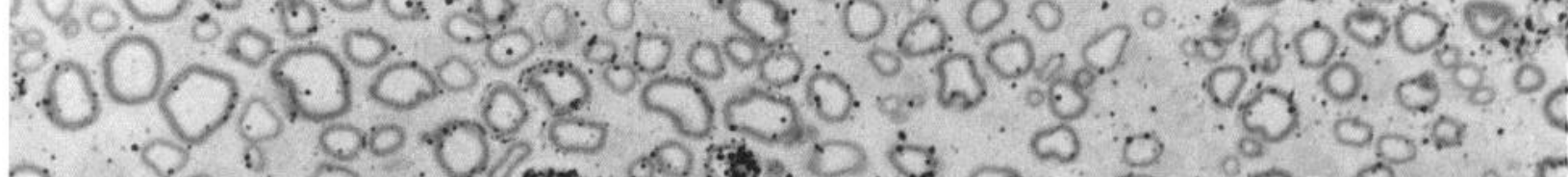

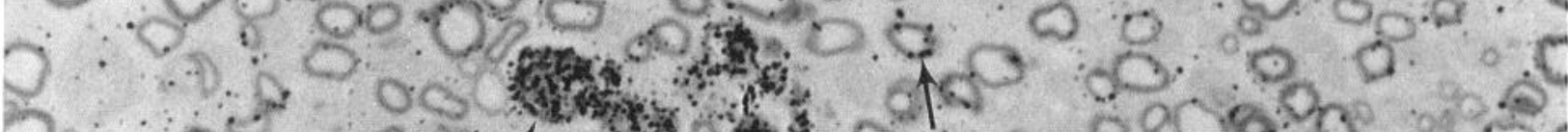

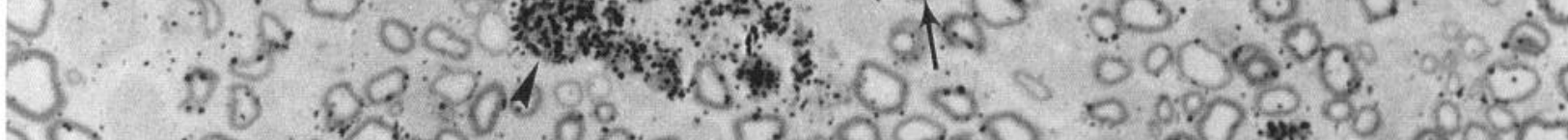

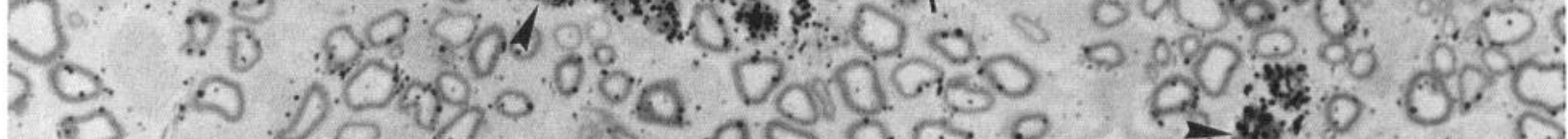
$800-00005090 \mathrm{C}$

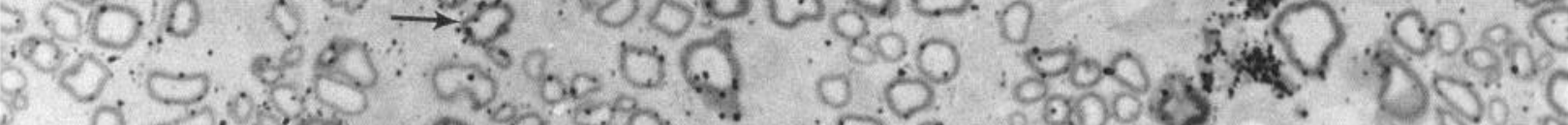

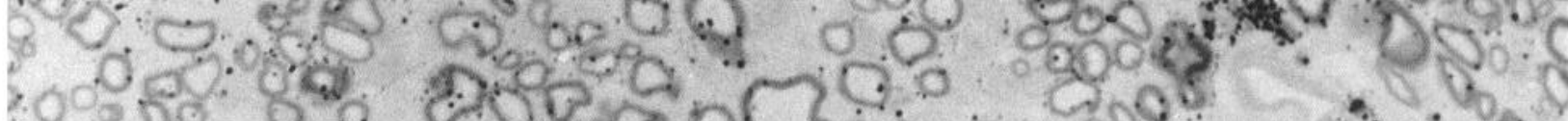

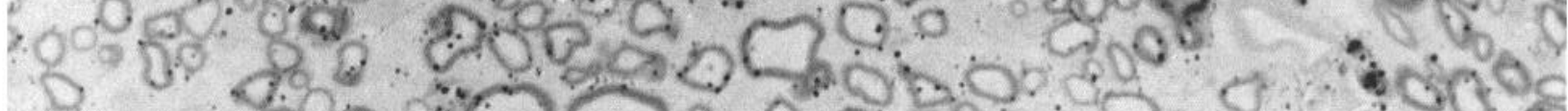

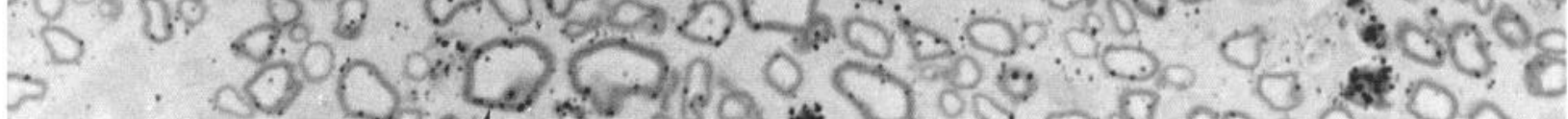

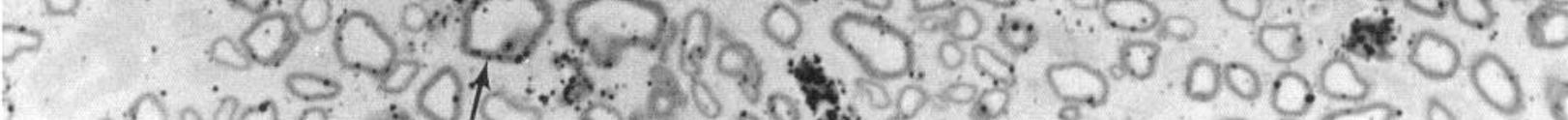
$0.000000 \%$ $200000000000 \%$ c $0000000000 \%$ 

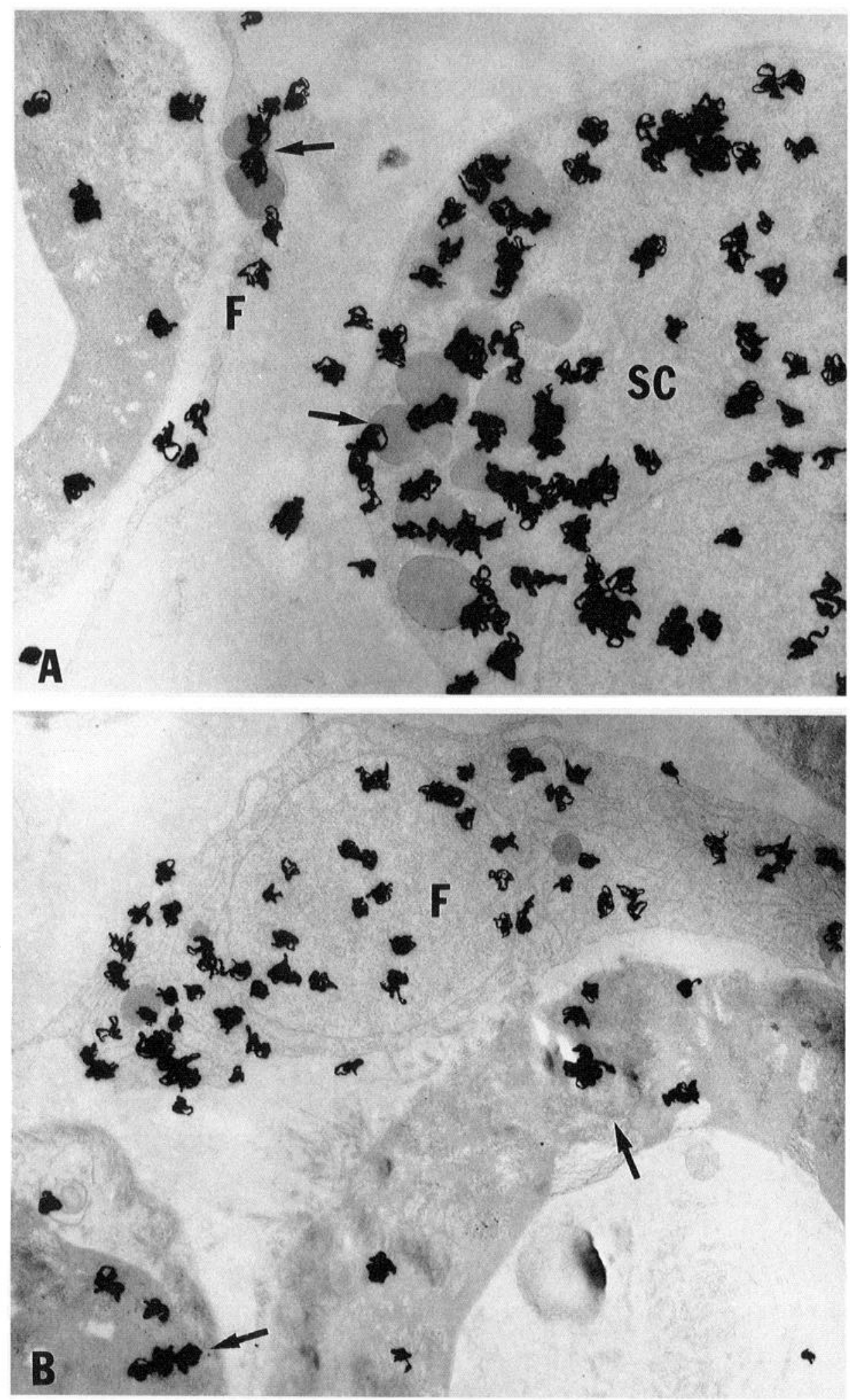

Figure 6. EM autoradiographs of sciatic nerve labeled with ${ }^{3} \mathrm{H}$-acetate $2 \mathrm{~d}$ after crush injury. A, Cytoplasm of Schwann cell $(S C)$ is heavily labeled. Lipid droplets in Schwann cells and fibroblasts $(F)$ are also labeled (arrows). Unstained section; $13,900 \times . B$, Cytoplasm of fibroblast $(F)$ is well labeled. Degenerating myelin also becomes labeled (arrows). Unstained section; $10,500 \times$.

Figure 5. Autoradiographs of sciatic nerve 4-10 weeks after nerve crush. $A$, Four weeks after nerve crush the new myelin sheaths are thick and well labeled. Unstained section; $5000 \times . B$, Extracellular labeled debris is present in the endoneurium at 10 weeks after crush. Unstained section; $6800 \times$. C, Six weeks after nerve crush endoneurial macrophages (arrowheads) still contain most of the labeled myelin debris. The myelin sheaths are also labeled (arrows). LM, unstained section; $700 \times$. 


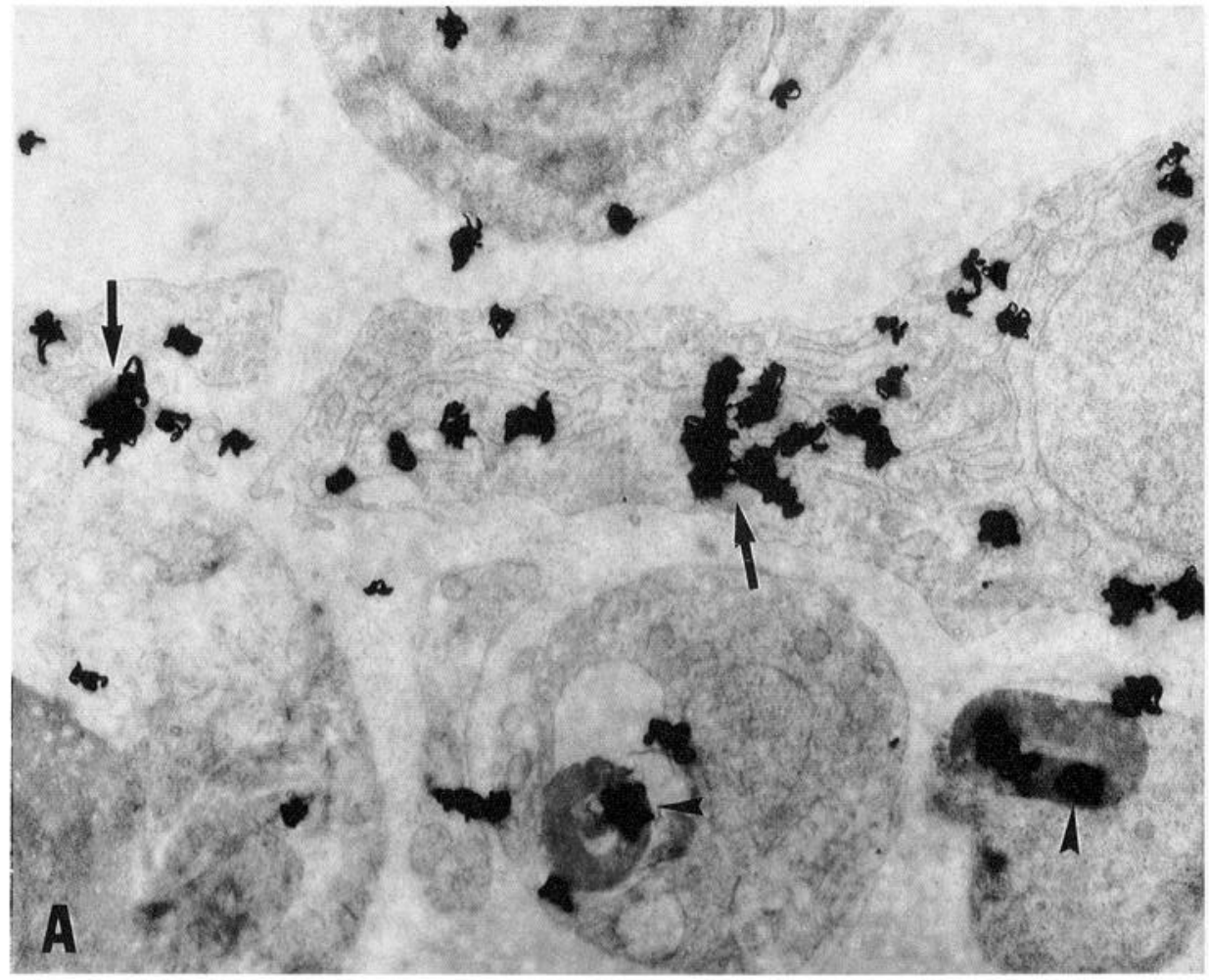

Figure 7. EM autoradiographs of sciatic nerve labeled with ${ }^{3} \mathrm{H}$-glycerol $2 \mathrm{~d}$ after crush injury. $A$, Lipid droplets in fibroblasts are well labeled (arrows). Degenerating myelin is also labeled $(a r$ rowheads). Unstained section; $10,900 \times$. $B$, Lipid droplets in Schwann cells (arrow) and myelin (arrowhead) are labeled. Unstained section; $11,000 \times$.

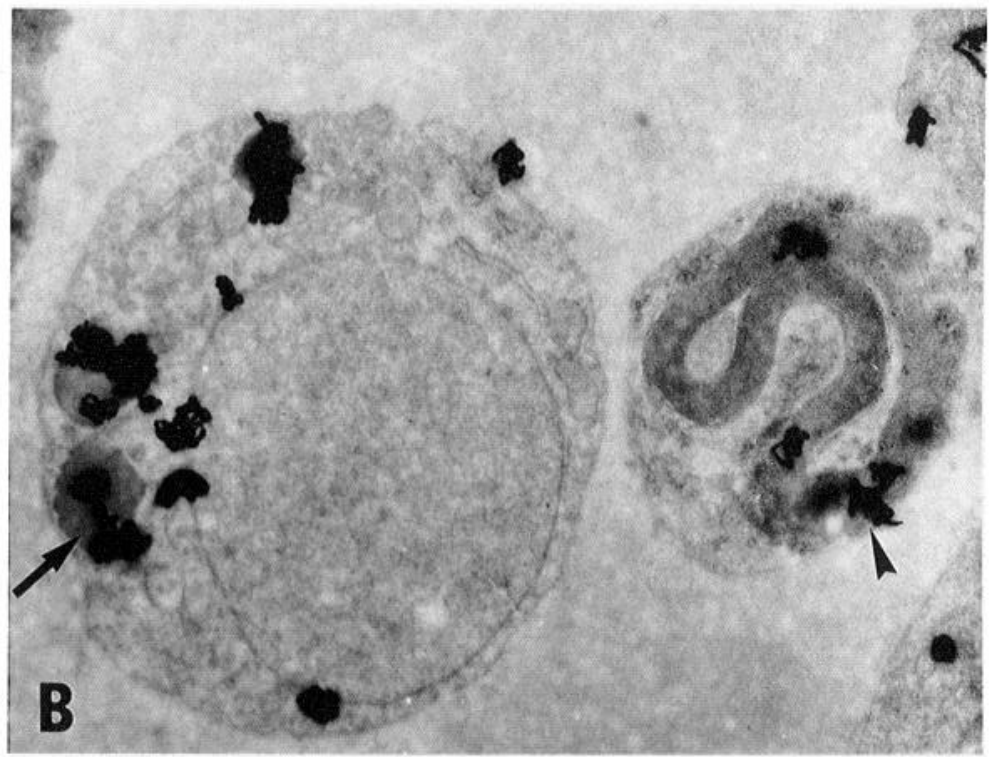

et al., 1989; Stoll et al., 1989). This interpretation is based in part on these droplets containing sudanophilic lipids (glycerolipids and cholesteryl esters). However, lipid droplets can arise in Schwann cells from newly synthesized lipid (Goodrum et al., 1990), raising the possibility that the lipid droplets appearing in Schwann cells shortly after a nerve crush reflect newly synthesized myelin lipids being routed into lipid droplets because myelin assembly is halting. When we used ${ }^{3} \mathrm{H}$-acetate to label the lipids synthesized de novo $2 \mathrm{~d}$ after nerve crush, the lipid droplets in Schwann cells became labeled, indicating that newly synthesized lipids do contribute to these structures. Moreover, when we used ${ }^{3} \mathrm{H}$-acetate to label myelin 4 weeks before nerve crush, the lipid droplets in Schwann cells after nerve crush again became labeled, indicating that degenerating myelin lipids also contribute to these structures, and supporting the contention that Schwann cells do catabolize some of their own myelin lipids. While we cannot rule out the possibility that there are two independent compartments of lipid droplets, one for newly synthesized lipids and the other for degenerating lipids, we suggest that these early-appearing lipid droplets represent a single storage compartment for both the excess of newly synthesized lipid that can no longer be routed to myelin and the lipid products of myelin degeneration.

Our labeling of lipids synthesized de novo at $2 \mathrm{~d}$ after nerve crush also revealed that some of the newly synthesized lipid still enters the myelin compartment, even to the extent of labeling 


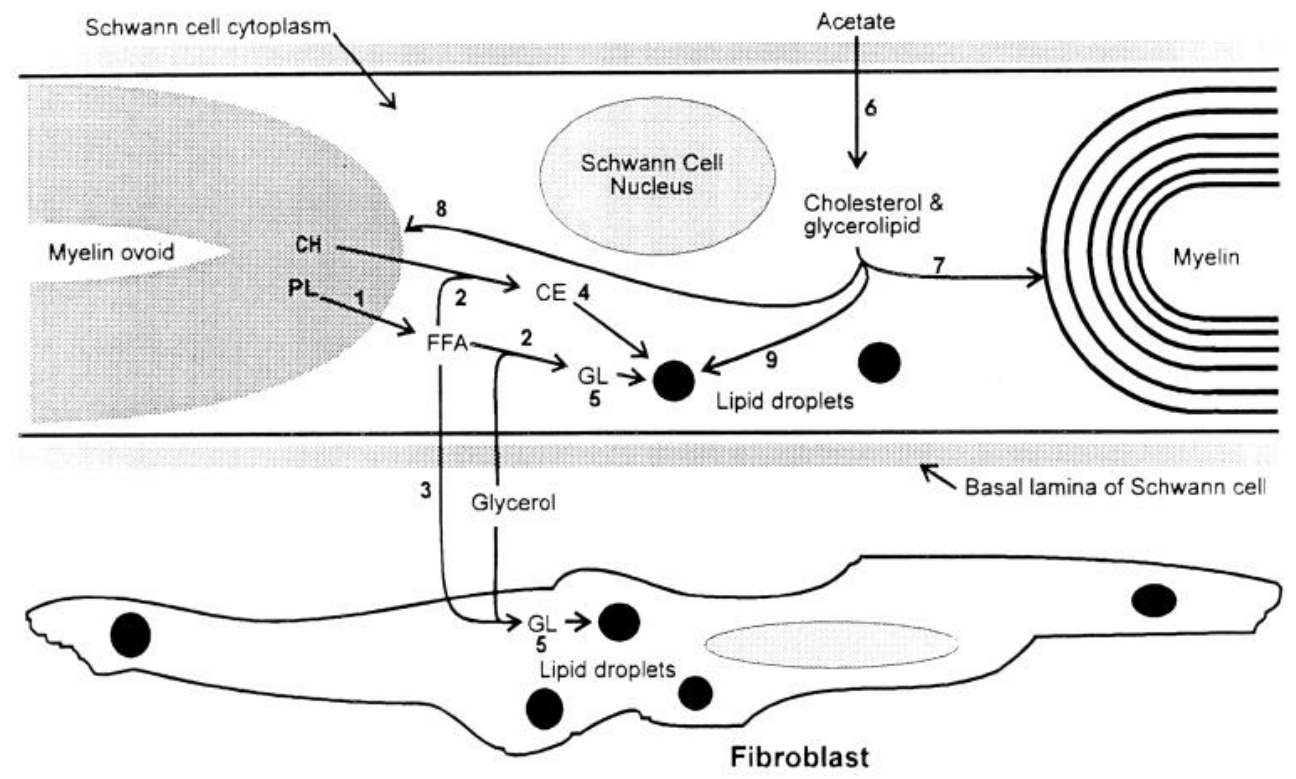

Figure 8. Cartoon of basal lamina-delimited Schwann-cell tube 2-3 d after nerve crush, illustrating the metabolic events described in the text. At this time axons have degenerated and the myelin has begun to break up into "myelin ovoids." As the myelin degenerates, phospholipids $(P L)$ are degraded by phospholipases to produce free fatty acids $(F F A)(1)$. These FFAs are recycled within the Schwann cell (2) or secreted and taken up by surrounding cells (3). FFAs are recycled through esterification reactions with cholesterol $(\mathrm{CH})$ or glycerol to produce cholesteryl esters $(C E)(4)$ and glycerolipids $(G L)(5)$. These products are then stored in lipid droplets. De novo synthesis of lipids (6) continues for the first few days after injury. The newly synthesized lipids are incorporated into myelin that still retains its periodicity (7), into myelin that has degenerated into ovoids ( 8 ), and into lipid droplets $(9)$. myelin that is in the process of breaking up into ovoids. These data indicate that for the first few days after nerve crush both myelin degeneration and new myelin assembly are occurring within the same cell.

There was a robust labeling of lipid droplets within fibroblasts and the resident macrophages by $3 \mathrm{~d}$ after crushing prelabeled nerves. This labeling must have resulted from the uptake of lipid breakdown products released into the extracellular space, since fibroblasts were never found within the Schwann-cell tubes. Lipid droplets in fibroblasts were also labeled by incubation of crushed nerve with lipid precursors. Glycerol, which is used in the recycling of fatty acids as well as in de novo lipid synthesis, was especially effective in labeling the fibroblasts' lipid droplets. These results are consistent with previous biochemical studies demonstrating that an increase in the esterification reactions utilizing glycerol is a very early event in Wallerian degeneration (White et al., 1989; Goodrum, 1990). White et al. (1989) postulated that the large pool of free fatty acids generated by the breakdown of myelin phospholipids promotes the increase in esterification reactions utilizing glycerol. Our data suggest that the fatty acids produced from the catabolism of myelin phospholipids are salvaged by being incorporated into glycerolipids within Schwann cells and fibroblasts (and resident endoneurial macrophages). The glycerolipids are then stored in lipid droplets within these cells. The lipid droplets subsequently disappear from Schwann cells and fibroblasts as regeneration proceeds, but the fate of these lipids is unknown. Figure 8 illustrates the proposed sequence of metabolic events occurring in Schwann cells and fibroblasts prior to macrophage infiltration.

\section{Myelin catabolism after macrophage infiltration}

One week after nerve crush, we found extratubal macrophages containing numerous lipid droplets but little myelin debris. Others have also reported similar findings (e.g., Boyles et al., 1989; Stoll et al., 1989). As described above, these lipid droplets probably arise from the esterification of free fatty acids released from the degenerating myelin into the extracellular space. There were, in contrast, much larger numbers of intratubal phagocytes and these were filled with labeled myelin debris. These intratubal phagocytes, which were frequently partially surrounded by
Schwann cells containing regenerating axons, have been identified as macrophages by immunohistochemistry (Stoll et al., 1989). By 2 weeks after nerve crush, extratubal macrophages were much more numerous, and they, like their intratubal counterparts at 1 week, now contained labeled myelin debris as well as labeled lipid droplets. No extracellular labeled myelin debris was seen at these times. We interpret these observations to indicate that, in the first week or so after nerve crush, most macrophages invade the Schwann-cell tubes to phagocytize the myelin debris. The later appearance of debris-laden extratubal macrophages represents the migration of macrophages out of the Schwann-cell tubes after they have phagocytized the myelin debris. This interpretation contrasts with that of Boyles et al. (1989), who consider all myelin debris within the basal lamina to be within Schwann cells, and to be completely resorbed by these Schwann cells. These authors attribute the later appearance of debris-laden extratubal macrophages to clearance of membrane debris in the extracellular space of the endoneurium. However, we found very little labeled extracellular myelin debris before 4 weeks, making their hypothesis unlikely. The small amounts of extracellular myelin debris we observed in crushed nerve from 4 weeks onward are suggested to arise from disintegrating macrophages (Boyles et al., 1989).

\section{Recycling of myelin cholesterol}

Our data confirm the early autoradiographic studies of Rawlins et al. (1970), 1972) demonstrating that myelin cholesterol is reincorporated into new myelin following Wallerian degeneration. While the present study labeled all myelin lipids rather than just cholesterol, cholesterol and cholesteryl esters account for $60-80 \%$ of the total label from $1 \mathrm{~d}$ to 10 weeks after nerve crush (see Goodrum, 1991, for the detailed time course and lipid distribution). Thus, to a large degree, the autoradiographs represent the distribution of myelin cholesterol in nerve.

Lipoproteins are hypothesized to have a major role in the salvage and reutilization of cholesterol in nerve. It was initially postulated that macrophage-derived apoE acquired cholesterol from the degenerating myelin and carried it to the macrophage for storage. From this storage site the cholesterol would be released in the form of lipoprotein particles to be taken up by the 
Table 1. Time course of biochemical and cellular events during Wallerian degeneration and nerve regeneration

Interval Event

1-3 DAYS De novo lipid synthesis rapidly declines; to near zero in the case of cholesterol.

Degradation of myelin phospholipids begins, generating a large pool of free fatty acids.

Free fatty acids produced by myelin degeneration are sequestered by esterification with glycerol and cholesterol in lipid droplets in Schwann cells, fibroblasts and macrophages. De novo synthesized lipids also enter the lipid droplets.

For a brief period, myelin assembly and myelin degradation occur simultaneously within the same cell.

3-6 DAYS Macrophages invade the degenerating nerve and rapidly penetrate the Schwann cell's basal lamina to phagocytize the degenerating myelin.

Apolipoproteins begin to appear in the nerve.

1 WEEK Regenerating axons begin to appear within the Schwann cell tubes.

There are many intratubal macrophages filled with myelin debris. Few extratubal macrophages are present, and these contain only lipid droplets generated from the free fatty acid pool.

2 WEEKS Macrophages, filled with myelin debris and lipid droplets, exit the Schwann-cell tubes.

There is a rapid rise in the endoneurial levels of apolipoproteins.

Thin myelin sheaths surround the regenerating axons. Schwann cells reutilize the degenerated myelin lipids provided by the macrophages via lipoproteins.

3-10 WEEKS Apolipoproteins A1, E, and D are at peak levels in nerve at $3-4$ weeks, declining thereafter to near control levels by 10 weeks.

Myelin sheaths continue to thicken and reutilize the degenerated myelin lipids.

De novo cholesterol synthesis in regenerating nerve remains at low levels for at least 10 weeks due to cholesterol reutilization via lipoproteins.

Numerous extratubal macrophages are present and are loaded with myelin debris. Some macrophages may eventually carry away the debris.

This table summarizes the proposed cellular and biochemical events related to myelin lipid disposition during Wallerian degeneration and the subsequent regeneration. This time course is based on the currently described data as well as the previously published work described in the Discussion.

myelinating Schwann cells (Mahley, 1988). More recently, several apolipoproteins, including some produced locally within the nerve (apoE, apoD) and others derived from the plasma (apoA-I, apoA-IV), have been postulated to play a role in the intraneural salvage and reutilization of cholesterol (Boyles et al., 1989, 1990; Spreyer et al., 1990).

The present data are relevant to several aspects of this process of cholesterol salvage and reutilization. First, based on the biochemical literature discussed above and the early labeling of
Schwann-cell lipid droplets demonstrated herc, it is apparent that Schwann cells begin to catabolize their own myelin lipids within a day following nerve crush. The early appearance of labeled myelin lipids in Schwann-cell lipid droplets clearly represents, in part, the recycling of free fatty acids by esterification of glycerol, as described above. Although it is not possible to determine from the present studies whether it is the preexisting myelin cholesterol or newly synthesized cholesterol that contributes to the formation of cholesteryl esters by the free fatty acids, we note that Rawlins et al. (1972) found prelabeled myelin cholesterol in lipid droplets of Schwann cells at $5 \mathrm{~d}$ after injury, and that we found labeled cholesteryl esters to increase at the expense of prelabeled myelin cholesterol (Goodrum, 1991). Thus, it appears that Schwann cells may initially salvage some of their own myelin cholesterol, as cholesteryl esters within lipid droplets (see Fig. 7), without the need for macrophages or lipoproteins. However, it is clear from the data presented here that the bulk of the myelin cholesterol comes to reside within macrophages as they phagocytize the myelin debris within the Schwanncell tubes.

Second, our conclusion that much of the myelin cholesterol comes to reside in macrophages through direct phagocytosis of the intratubal myelin debris suggests that lipoproteins do not transport cholesterol from the Schwann cell's degenerating myelin sheath to the extratubal macrophage for storage as initially hypothesized. Instead, the data presented here suggest that lipoproteins in injured nerve are primarily involved in the regeneration of nerve, that is, in carrying cholesterol from the extratubal macrophages to the intratubal Schwann cells myelinating the regenerating axons. This interpretation is consistent with the observations that the peak levels of most apolipoproteins, as well as of lipoprotein-bound myelin cholesterol in nerve, occur 2-4 weeks after crush injury (Boyles et al., 1990; Goodrum, 1991).

Third, our finding that a large amount of labeled myelin lipid persists as debris in macrophages 10 weeks after injury suggests that the potential for reutilization of cholesterol may be present for several months. This observation is consistent with our previous report that cholesterol synthesis in regenerating nerve is depressed for up to 15 weeks (Goodrum, 1990). On the other hand, the levels of apolipoproteins in regenerating nerve are nearing control levels by 10 weeks (Boyles et al., 1990), making significant lipoprotein-mediated reutilization of cholesterol unlikely after 10 weeks. Thus, the eventual fate of this persistent myelin debris is unknown. Stoll et al. (1989) observed foamy macrophages near endoneurial and perineurial vessels, and suggested that these macrophages were leaving the nerve. We also saw labeled macrophages adjacent to endoneurial vessels, but, as is evident in Figure $5 B$, most of the macrophages were not adjacent to endoneurial vessels at the time points we examined. While preliminary biochemical data suggest that most, if not all, the labeled myelin cholesterol is retained within the nerve for up to 12 weeks (Goodrum, 1991), we cannot rule out the possibility that macrophages eventually carry away a significant portion of the myelin cholesterol.

A summary of our proposed time course of the biochemical and cellular events related to myelin lipids during Wallerian degeneration and nerve regeneration is provided in Table 1.

\section{References}

Beuche W, Friede RL (1984) The role of nonresident cells in Wallerian degeneration. J Neurocytol 13:767-796. 
Boyles JK, Zoellner CD, Anderson LJ, Kosik LM, Pitas RE, Weisgraber KH, Hui DY, Mahley RW, Gebicke-Haerter PJ, Ignatius MJ, Shooter EM (1989) A role for apolipoprotein E, apolipoprotein A-1, and low density lipoprotein receptors in cholesterol transport during regeneration and remyelination of the rat sciatic nerve. J Clin Invest 83:1015-1031.

Boyles JK, Notterpek LM, Anderson LJ (1990) Accumulation of apolipoproteins in the regenerating and remyelinating mammalian peripheral nerve. J Biol Chem 265:17805-17815.

Crang AJ, Blakemore WF (1986) Observations on Wallerian degeneration in explant cultures of cat sciatic nerve. J Neurocytol 15:471482.

Gelman BB, Goodrum JF, Bouldin TW (1991) Macrophage apolipoprotein synthesis and endoneurial distribution as a response to segmental demyelination. J Neuropathol Exp Neurol 50:383-407.

Goodrum JF (1990) Cholesterol synthesis is down-regulated during regeneration of peripheral nerve. J Neurochem 54:1709-1715.

Goodrum JF (1991) Cholesterol from degenerating nerve myelin becomes associated with lipoproteins containing apolipoprotein E. J Neurochem 56:2082-2086.

Goodrum JF, Earnhardt TS, Goines ND, Bouldin TW (1990) Lipid droplets in Schwann cells during tellurium neuropathy are derived from newly synthesized lipid. J Neurochem 55:1928-1932.

Goodrum JF, Farnhardt TS, Goines ND, Bouldin TW (1993) Fate of myelin lipids during nerve degeneration. J Neuropathol Exp Neurol 52:297.

Gould RM, Holshek J, Silverman W, Spivack WD (1987) Localization of phospholipid synthesis to Schwann cells and axons. J Neurochem 48:1121-1131.

Ignatius MJ, Gebicke-Haeter PJ, Skene JHP, Schilling JW, Weisgraber KH, Mahley RW, Shooter EM (1986) Expression of apolipoprotein E during degeneration and regeneration. Proc Natl Acad Sci USA 83: $1125-1129$

Mahley RW (1988) Apolipoprotein E: cholesterol transport protein with expanding role in cell biology. Science 240:622-630.
Napolitano LM, Scallen TJ (1969) Observations on the fine structure of peripheral nerve myelin. Anat Rec 163:16.

Popko B, Goodrum JF, Bouldin TW, Zhang SH, Maeda N (1993) Nerve regeneration occurs in the absence of apolipoprotein $\mathrm{E}$ in mice. J Neurochem 60:1155-1158.

Rawlins FA, Hedley-White ET, Villegas G, Uzman BG (1970) Reutilization of cholesterol-1,2- ${ }^{3} \mathrm{H}$ in the regeneration of peripheral nerve. Lab Invest 22:237-240.

Rawlins FA, Villegas G, Hedley-White ET, Uzman BG (1972) Fine structural localization of cholesterol-1,2- ${ }^{3} \mathrm{H}$ in degenerating and regenerating mouse sciatic nerve. J Cell Biol 52:615-625.

Salpeter MM (1981) High resolution autoradiography. In: Techniques in cellular physiology, Pt 1, pp 1-45. New York: Elsevier.

Salpeter MM, McHenry FA, Salpeter EE (1978) Resolution in electron microscope autoradiography. IV. Application to analysis of autoradiographs. J Cell Biol 76:127-145.

Scallen TJ, Dietert SE (1969) The quantitative retention of cholesterol in mouse liver prepared for electron microscopy by fixation in digitonin-containing aldehyde solution. J Cell Biol 40:802-813.

Shubert T, Friede RL (1981) The role of endoneurial fibroblasts in myelin degradation. J Neuropathol Exp Neurol 40:134-154.

Snipes GJ, McGuire CB, Norden JJ, Freeman JA (1986) Nerve injury stimulates the secretion of apolipoprotein $\mathrm{E}$ by non-neuronal cells. Proc Natl Acad Sci USA 83:1130-1134.

Spreyer P, Schaal H, Kuhn G, Rothe T, Unterbeck A, Olek K, Müller HW (1990) Regeneration-associated high level expression of apolipoprotein $\mathrm{D}$ mRNA in endoneurial fibroblasts of peripheral nerve. EMBO J 9:2479-2484.

Stoll G, Griffin JW, Li CY, Trapp BD (1989) Wallerian degeneration in the peripheral nervous system: participation of both Schwann cells and macrophages in myelin degradation. J Neurocytol 18:671-683.

White FV, Toews AD, Goodrum JF, Novicki DL, Bouldin TW, Morell $P$ (1989) Lipid metabolism during early stages of Wallerian degeneration in the rat sciatic nerve. J Neurochem 52:1085-1092. 